DOI: 10.17951/ff.2018.36.1.87-96

A N N A LES

UNIVERSITATIS MARIAE CURIE-SKŁODOWSKA

LUBLIN - POLONIA

VOL. XXXVI

SECTIO FF

$1-2018$

WANDA FIJAŁKOWSKA

ORCID ID: https://orcid.org/0000-0002-4161-6667

Uniwersytet Warszawski

\title{
La comparaison des dérivés français et polonais comme un test d'universalité d'une technique de construction de mots
}

Analiza porównawcza derywatów francuskich i polskich jako test uniwersalności techniki słowotwórczej

Comparing Polish and French derivates as a test of universality for a word-formation technique

Encore à neuf ans d'ici, les auteurs des ouvrages traitant de la formation de mots distinguaient cinq techniques de base: la suffixation, la préfixation, la conversion $^{1}$, la postfixation, la siglaison plus une sixième méthode, alliant au moins deux radicaux - la composition. D'aucuns mentionnaient la parasynthèse, la troncation ${ }^{2}$, la dérivation par échange de suffixes, et, au sein de la composition, les zrosty en polonais (le terme correspondant français, composés lexicaux unifiés, n'est pas utilisé fréquemment).

Cet état de choses se voit entre autres dans les ouvrages de Renata Grzegorczykowa et Jadwiga Puzynina (Grzegorczykowa, 1972), (Puzynina, 1978a; Puzynina, 1978b), (Grzegorczykowa and Puzynina, 1979), (Grzegorczykowa and Puzynina, 1998), Bogusław Kreja (Kreja, 1963 ; Kreja, 1972), Krystyna Kallas (Kallas, 1998), Hanna Jadacka (Jadacka, 2001 ; Jadacka, 2005; Jadacka, 2007), Alicja Nagórko (Nagórko, 1998; Nagórko, 2010), Mirosław Skarżyński (Skarżyński, 1999; Skarżyński, 2003a; Skarżyński, 2003b), Krystyna Waszakowa (Waszakowa, 1991; Waszakowa, 1993), et, tout récemment, d’Iwona Kaproń-Charzyńska

${ }^{1}$ Rappelons que la formation de noms déverbaux du type $:$ bondir $\rightarrow$ bond, galoper $\rightarrow$ galop, communément classés comme des cas de dérivation régressive, sont en fait des cas de conversion.

${ }^{2}$ Ou dérivation régressive, p.ex. professeur $\rightarrow$ prof, cinématographe $\rightarrow$ cinéma. 
(Kaproń-Charzyńska, 2005) et de Renata Przybylska (Przybylska, 2006) - pour ce qui est de la langue polonaise.

Cette conception de l'ensemble des techniques de construction de mots n'a changé qu'au moment où les chercheurs polonais ont découvert un groupe assez nombreux de dérivés dont l'apparition ne se laissait expliquer autrement que par l'échange de préfixes.

Une première suggestion que certains dérivés classés comme préfixés simples ne le sont pas fut énoncée par Mirosław Skarżyński en 2004 dans Wstęp [Introduction] du Stownik gniazd stowotwórczych ${ }^{3}$; ont succédé à cette déclaration des analyses-sondages menées par Joanna Stankiewicz (Stankiewicz, 2006). Enfin furent publiés trois travaux de Michał Wiśnicki, les articles de 2006 et de 2009 (Wiśnicki, 2006 ; Wiśnicki, 2009), puis sa thèse de doctorat de 2010, Derywacja prefiksalna wymienna $w$ czasownikach polskich (na materiale gniazdowym). L'auteur y a rendu compte de ses recherches sur un certain nombre de dérivés de verbes accomplis polonais, menées à l'aide d'outils fournis par la méthode «nidologique» [gniazdowa]. Ses recherches ont abouti à la découverte de verbes accomplis créés par échange de préfixes: une technique de création qui n’a été ni décrite, ni signalée auparavant. L'auteur de cette monographie a défini cette technique comme suit:

La dérivation par échange de préfixes consiste à éliminer le préfixe d'un verbe accompli (divisible selon les lois de la formation de mots) et le remplacer par un autre préfixe dans le verbe accompli motivé. [trad. W.F.].

Cette méthode de dérivation est illustrée entre autres par les verbes: ubrać się $\rightarrow$ rozebrać się (et non *rozeubrać się), zbudować $\rightarrow$ odbudować (et non *odzbudować), narysować $\rightarrow$ przerysować (et non *przenarysować), zakodować $\rightarrow$ odkodować (et non *odzakodować), uktonić się $\rightarrow$ odktonić się (et non *oduktonić się), wprowadzić $\rightarrow$ wyprowadzić (et non point *wywprowadzić) $)^{4}$.

Il convient de souligner l'importance du caractère accompli des bases des dérivés formés par échange de préfixes (désormais DÉP). En effet, elle assure la consécution logique des actions dénotées : une construction non détruite auparavant ne peut être odbudowana (rebâtie), et l'on ne peut détruire que ce qui a été zbudowane (bâti). De même, on ne peut resserrer un boulon qui n'a pas été desserré.

Pourtant ce type de dérivés pose une difficulté d'interprétation: si la base de chacun d'entre eux est préfixée, comment prouver que c'est cette base entière qui

3 «Les verbes déverbaux que l'on peut classer comme formés par échange de préfixes sont plus de trois cents, mais il semble que l'étendue des phénomènes qui se laissent interpréter de la sorte est bien plus grande.» [Skarżyński, 2004, XII] [trad. W.F.].

${ }^{4}$ La polypréfixation (concaténation des préfixes) existe bien en polonais comme en français, p.ex. nawypisywać, poprzenosić, zrepolonizować; réarranger, rembourser, désendetter. 
motive le DÉP, et non le verbe simple, si le préfixe en question n'est pas incorporé dans le produit? Il s'avère que les morphologues disposent à cette fin d'un excellent outil, défini il y a plusieurs dizaines d'années par J. Puzynina, la paraphrase constructionnelle. Correctement formulée, elle explique la signification du mot construit de manière la plus simple possible en se référant directement au mot motivant. Ainsi, dobudować 'zbudować coś dodatkowo', surgeler 'congeler très fort' prouvent leur formation à base de verbes préfixés, et partant leur statut de DÉP.

Les dérivés créés par échange de préfixes une fois dégagés de parmi les verbes accomplis polonais, on a pu en faire l'analyse, tant morphologique que sémantique. Il s'est avéré que cette technique occupe une place importante dans le système polonais de construction de mots, et fait montre d'une grande diversité formelle et sémantique, et les préfixes engagés sont fortement spécialisés dans leur instruction. Une lacune dans la description des verbes polonais fut ainsi comblée: la dérivation des verbes ne se limite ni à une affixation simple, ni à la conversion (ni aux deux procédures appliquées à la fois).

Ces affirmations étaient pertinentes, mais au cours du temps la valeur scientifique des thèses portant sur l'échange de suffixes diminuait. On pouvait craindre que l'échange d'affixes en général perdra sa place dans le polonais actuel et sa description.

Pour élucider ces doutes, on a entrepris une recherche pour actualiser le matériel collecté. L'actualisation chronologique devait vérifier si le polonais multiplie les cas de DÉP ou si c'est une procédure en voie d'extinction. L'actualisation des sources avait pour but de fournir des preuves de l'éventuelle activité de cette technique dans le polonais vivant, présent dans les corpus et sur Internet. Les dictionnaires qui ont alimenté les recherches antérieures faisaient appel surtout aux textes de polonais général, en majorité littéraires, souvent anciens.

Les résultats obtenus ont confirmé la supposition que le nombre de DÉP en polonais augmente, et documenté la présence de ce type de verbes dans le polonais courant actuel (voir odesemesować, odmailować). Partant, ils nous ont aussi incitée à poser une question suivante: la dérivation par échange de préfixes est-elle une technique endémique polonaise? Peut-on la retrouver dans d'autres langues à genèse différente? Nous avons choisi le français pour cette confrontation, l'estimant un objet approprié d'études.

Dans la littérature morphologique traitant du français sont citées le plus souvent les mêmes procédures principales de formation de mots que dans les ouvrages portant sur le polonais: la suffixation, la préfixation, la troncation (surtout l'apocope), la parasynthèse, la conversion, la composition. Ce techniques figurent dans les manuels de H. Huot (Huot, 2006), de Denis Apothéloz (Apothéloz, 2002), d'Hervé Béchade (Béchade, 1994), de Dominique Maingueneau, Jean-Louis Chiss 
et Jacques Filliolet (Maingueneau, Chiss and Filliolet, 2013), et ont fait l'objet d'analyses approfondies de Danielle Corbin (Corbin, 1987; Corbin, 2001), Bernard Fradin, Françoise Kerleroux et Marc Plénat (Fradin, Kerleroux and Plénat, 2009), Georgette Dal (Dal, 2001), Hélène Huot (Huot, 2007), et bien d'autres. A ce noyau dur viennent s'ajouter les procédures dites marginales, décrites notamment par Bernard Fradin (Fradin, 2009), Michel Roché (Roché, 2008: 215), et les techniques estimées extragrammaticales, dont la formation de mots-valises, les langages secrets, tels le verlan, le javanais, et enfin la création d'hypocoristiques par redoublement. La suffixation substitutive est mentionnée pour la première fois en 2005 par Geert Booij (Booij, 2005 : 13, cité par Fradin et al., 2009: 15). En revanche, bien que d'amples ouvrages aient été consacrés aux préfixes - telles les Recherches sur la suffixation dans le français contemporain de Jean Peytard (Peytard, 1975), les études de Dany Amiot (Amiot, 2002; Amiot, 2008) Pierre Jalenques (Jalenques, 2002; Jalenques, 2014), de Denis Apothéloz (Apothéloz, 2007a; Apothéloz, 2007b), de Robert Martin (Martin, 2004) et de Francine Gerhard-Krait (Gerhard-Krait, 2000), pour ne mentionner que les œuvres les plus connues, la substitution de préfixes n'est que timidement signalée dans le Trésor de la langue française, sans développement théorique.

Nous nous sommes donc posé, dans un premier temps, la question si cette technique, manifestement fréquente en polonais, est utilisée en français. En 2010, nous avons pu y répondre positivement; en partant d'un premier exemple - se remaquiller construit sur se démaquiller, nous avons collecté environ 200 verbes construits par échange de préfixes, comme p.ex. accrocher - décrocher, empoter-dépoter, décarreler - recarreler. Là aussi, la paraphrase constructionnelle a prouvé son utilité pour vérifier les rapports entre les dérivés et les bases supposées: décrocher 'enlever qch qui a été auparavant accroché', dépoter 'sortir d'un pot qch qui a été empoté', recarreler 'remettre le carrelage sur qch qui a été décarrelé', détuber 'enlever le tube à qqn qui a été intubé', resserrer 'serrer de nouveau ce qui a été desserré'.

Dans un second temps, nous avons entrepris une recherche approfondie (Fijałkowska, 2016), pour mieux cerner l'étendue de cette procédure et vérifier la pertinence du classement sémantique de Wiśnicki en dix groupes - distributifs, annulatifs, supplémentaires, intensifs, restauratifs, transformatifs, répétitifs, méthodiques, de direction d'action, réactifs - applicable, au premier coup d'œil, au français comme au polonais.

Soulignons ici que la présente recherche sur la dérivation par échange de préfixes porte sur un fragment du système morphologique des deux langues, et non le total du système lexical. Nous avons abordé ce problème en étant consciente du fait que le système polonais de construction de mots est bien plus développé que 
le système français; les différences en nombre des marqueurs morphologiques des DÉP, en faveur du polonais, seront traités comme évidentes. Ce qui est soumis à l'étude, c'est l'échelle de l'application de cette technique.

Les dérivés verbaux formés moyennant cette technique se sont avérés nombreux en français; si l'on ajoute aux lexèmes attestés ceux qui apparaissent sur Internet en nombre supérieur à 20 occurrences, le chiffre en monte bien au-dessus de quatre centaines. À la différentiation formelle - l'emploi de $\boldsymbol{d e}$-. re-, $\boldsymbol{e x}$-, trans-, $r \boldsymbol{e}_{-}$, sur- s'ajoute une diversité sémantique; en effet, on distingue:

- des verbes annulatifs, de loin le plus nombreux, tels débarrasser, débourrer, débuer, dégourdir, se dékyster, dépoussiérer, détuber, disjoindre,

- des verbes effectifs, tel p.ex. surgeler (provenant de congeler),

- des verbes restitutifs, tels recroiser, reloger, remeubler, renationaliser, retordre,

- des verbes transformatifs, p.ex. transmettre.

Les recherches menées sur Internet se sont avérées fructueuses sous plus d'un égard. Nous avons rassemblé presque deux centaines de lexèmes DÉP. Qui plus est, ces lexèmes, classés par nombre d'occurrences, se sont avérés appartenir aux divers strates du français:

- depuis le langage général, de tous les jours - comme se revêtir, se réinstaller, découver, démurer, se démerder, dé(s)sabler, dégrener - avec une fréquence de plus de 5000;

- fréquent, tels défermer, désorceler, défouir, détuber, se recoiffer, allant de 501 à 5000 occurrences;

- périphérique, de 100 à 500 occurrences, p.ex. débouer, désacher;

- et enfin marginal, comme déglober, dénuager, déplatir, se recalcifier, repoissonner n'atteignant qu'au-dessous de 50 occurrences.

La vitalité de l'échange des préfixes en français ne se manifeste pas uniquement par le nombre de verbes recensés en dehors des dictionnaires et leur diversification sémantique. Quelques dizaines de verbes DÉP ont servi à leur tour de bases pour des dérivés du même type: en d'autres termes, nous nous trouvons en présence de trios de verbes liés par motivation successive, dont le second et le troisième sont formés par échange de préfixes. C'est le cas notamment de:

- emballer $\rightarrow$ déballer $\rightarrow$ remballer,

- encadrer $\rightarrow$ décadrer $\rightarrow$ recadrer,

- encager $\rightarrow$ décager $\rightarrow$ recager,

- encercler $\rightarrow$ décercler $\rightarrow$ recercler (un tonneau),

- enclore $\rightarrow$ déclore $\rightarrow$ reclore,

- $s$ 'entortiller $\rightarrow$ se détortiller $\rightarrow$ se retortiller. 
Le fait que non seulement certains DÉP figurent dans le système du français d'aujourd'hui, mais qu'ils servent à leur tour de bases à d'autres DÉP, témoigne de ce que cette technique y fonctionne à pleine capacité. Le français, connu pour être bien plus analytique et moins «constructeur de mots» en général que le polonais, montre ici une face toute nouvelle.

$\mathrm{Au}$ vu de l'étude menée présentement sur les DÉP, les distinctions entre le polonais et le français paraissent plus quantitatives que qualitatives. La technique d'échange de préfixes est employée dans les deux langues à grande échelle, et les produits de cette dérivation se laissent classer en plusieurs groupes sémantiques. Elle fait montre d'une activité forte, stable, ce qui se traduit, outre un nombre fort de dérivés, 1. par la présence des lexèmes verbaux DÉP non attestés sur Internet, 2. par leur appartenance à toutes les couches lexicales - des unités du langage fréquent jusqu'aux créations marginales, 3. par la création de DÉP de deuxième ordre (à partir de DÉP issus, eux, de V simples).

\section{CONCLUSIONS}

Une telle comparaison, tout d'abord, permet de voir plus précisément les systèmes morphologiques des deux langues, et surtout permet d'affirmer que distinguer encore une technique de construction de mots n'est pas un abus méthodologique, mais constitue une procédure de recherche fiable, dont les résultats semblent avoir une certaine valeur en ce domaine de la linguistique. La comparaison menée jusquelà nous laisse supposer que la dérivation par échange de préfixes, qui fonctionne dans des langues de familles différentes, est aussi un élément non négligeable des systèmes d'autres langues à morphologie comparable. Pour que cette supposition devienne certitude, il faudrait élargir le champ de recherche, p.ex. en suivant la voie ouverte par L.I. Roïzenzon (Roïzenzon, 1974) - celle de recherches sur la polypréfixation dans les langues slaves. Ses résultats peuvent être considérés sous un autre angle, prenant en compte les DÉP.

Puisque la dérivation par échange de préfixes n'est nullement une technique marginale, en voie d'extinction ni restreinte quant au niveau de langue, elle mérite ses droits de cité en morphologie, et la description des systèmes respectifs devrait être modifiée de sorte à lui assurer une position convenable. 


\section{RÉFÉRENCES BIBLIOGRAPHIQUES}

Amiot, D. (2002). Re-, préfixe aspectuel. Cahiers Chronos, 10, 1-20.

Amiot, D. (2008). La catégorie de la base dans la préfixation en dé-. In: B. Fradin (éd.), La raison morphologique. Hommage à la mémoire de Danielle Corbin (pp. 1-15). Amsterdam/Philadelphia: Benjamins.

Apothéloz, D. (2002). La construction du lexique français : principes de morphologie dérivationnelle. Ophrys: L'Essentiel français.

Apothéloz, D. (2007a). Procès centrifuge et procès centripète: RE-, DÉS- et l'antonymie directionnelle en français. In: N. Hathout, F. Montermini (eds.), Morphologie à Toulouse. Actes du colloque international de Morphologie « $4^{\text {èmes }}$ Décembrettes» (pp. 149-169). München: Lincom Europa (LSTL).

Apothéloz, D. (2007b). La préfixation en RE-, l'antonymie directionnelle et les phénomènes de polarité sémantique. Journal of French Language Studies, 17, 143-158.

Béchade, H. (1994). Grammaire française. Paris: PUF.

Booij, G. (2005). The Grammar of Words. An Introduction to Linguistic Morphology. Oxford: Oxford University Press.

Corbin, D. (1987). Morphologie dérivationnelle et structuration du lexique, Thèse de doctorat d'État soutenue le 7 janvier 1987 à l'Université de Paris VIII. L'information grammaticale. Https://doi.org/10.3406/igram.1987.2091

Corbin, D. (2001). Préfixes et suffixes. Du sens aux catégories. Journal of French Language Studies, $11,41-69$.

Dal, G. (2001). Contre préposition, contre préfixe. Recherches Linguistiques de Vincennes, 26, $173-201$.

Fijałkowska, W. (2010). La substitution de préfixes comme méthode de dérivation de verbes. In: A. Dutka-Mańkowska, T. Giermak-Zielińska (eds.), Des mots et du texte aux conceptions de la description linguistique (pp. 157-165). Warszawa: Wydawnictwa Uniwersytetu Warszawskiego.

Fijałkowska, W. (2016). La dérivation par échange de préfixes en tant que problème de recherche en linguistique contrastive. Kwartalnik Językowy, 63(4), 500-506.

Fradin, B., Kerleroux, F., Plénat, M. (eds.). (2009). Aperçus de morphologie du français. Saint-Denis : Presses Universitaires de Vincennes.

Gerhard-Krait, F. (2000). La Préfixation en dé(s)-: formes construites et interprétations. Thèse: Sciences du langage: Strasbourg 2.

Grzegorczykowa, R. (1972). Zarys stowotwórstwa polskiego. Stowotwórstwo opisowe, Warszawa: Wydawnictwa Uniwersytetu Warszawskiego.

Grzegorczykowa, R., Puzynina, J. (1979). Słowotwórstwo współczesnego języka polskiego. Rzeczowniki sufiksalne rodzime. Warszawa: Państwowe Wydawnictwo Naukowe.

Grzegorczykowa, R., Laskowski, R., Wróbel, H. (eds.). (1998). Gramatyka współczesnego języka polskiego. Morfologia. Warszawa: PWN.

Grzegorczykowa, R., Puzynina, J. (1998). Problemy ogólne słowotwórstwa. In: R. Grzegorczykowa, R. Laskowski, H. Wróbel (eds.), Gramatyka współczesnego języka polskiego. Vol. 2: Morfologia (pp. 361-388). Warszawa: PWN.

Huot, H. (2006). La morphologie. Forme et sens des mots du français. Paris : Colin.

Huot, H. (2007). La Préfixation négative en français moderne. In: F. Floricic (ed.), La Négation dans les langues romanes (pp. 177-203). Amsterdam/Philadelphia: John Benjamins.

Jadacka, H. (2001). System stowotwórczy polszczyzny (1945-2000). Warszawa: PWN.

Jadacka, H. (2005). Kultura języka polskiego. Fleksja, słowotwórstwo, składnia. Warszawa: PWN. 
Jadacka, H. (2007). O hierarchizacji w słowotwórstwie i jej skutkach dla opisu systemu derywacyjnego (na marginesie książki Iwony Kaproń-Charzyńskiej Derywacja ujemna w języku polskim. Rzeczowniki i przymiotniki). LingVaria, 1(3), 9-20.

Jalenques, P. (2002). Étude sémantique du préfixe RE en français contemporain: à propos de plusieurs débats actuels en morphologie dérivationnelle. Langue française, 133(1), 74-90.

Jalenques, P. (2014). Le problème de l'opacité sémantique dans les verbes préfixés en DÉ-. Pour une approche sémantique constructiviste. In: [actes de] Congrès Mondial de Linguistique Française, (pp. 1775-1796).

Kallas, K. (1998) . Przymiotnik. In: R. Grzegorczykowa, R. Laskowski, H. Wróbel (eds.), Gramatyka wspótczesnego języka polskiego. Morfologia (pp. 469-521). Warszawa: PWN.

Kaproń-Charzyńska, I. (2005). Derywacja ujemna w języku polskim. Rzeczowniki i przymiotniki. Toruń: Wydawnictwo UMK.

Kreja, B. (1963). Pojęcie derywacji wymiennej. Z polskich studiów slawistycznych. Seria 2. Językoznawstwo, 1, 33-140.

Kreja, B. (1972). Z zagadnień analizy słowotwórczej. In: J. Puzynina (ed.), Problemy wspótczesnego stowotwórstwa a dydaktyka uniwersytecka (pp. 59-78). Warszawa: Wydawnictwa UW.

Kreja, B. (1978). O antonimicznej kategorii słowotwórczej czasowników inceptywno-anulatywnych. Z zagadnień ogólnych polskiego słowotwórstwa. Studia, vol. 3, 307-317.

Maingueneau, D., Chiss, J.-L., Filliolet, J. (2013). Introduction à la linguistique française, tome 1 : Notions fondamentales, phonétique, lexique. Vanves: Hachette.

Martin, R. (2004). Le couple préfixal en-(in-) / es-(e-/ex-) en moyen français, Romania, 122(485-486), $1-45$.

Nagórko, A. (1998). Zarys gramatyki polskiej (ze słowotwórstwem). Warszawa: PWN.

Nagórko, A. (2010). Podręczna gramatyka języka polskiego. Warszawa: PWN.

Peytard, J. (1975). Recherches sur la préfixation en français contemporain. Paris: Champion.

Przybylska, R. (2006). Schematy wyobrażeniowe a semantyka polskich prefiksów czasownikowych do-, od-, prze-, roz-, u-. Kraków: Universitas.

Puzynina, J. (ed.). (1972). Problemy współczesnego stowotwórstwa a dydaktyka uniwersytecka. Warszawa: Wydawnictwa UW.

Puzynina, J. (1978 a). Próba klasyfikacji formantów słowotwórczych języka polskiego. Język Polski, $2,98-107$.

Puzynina, J. (1978 b). Typy formantów słowotwórczych w językach słowiańskich. Z polskich studiów slawistycznych, 4, 179-188.

Roché, M. (2008). Quelques exemples de morphologie non conventionnelle. In: B. Fradin (ed.), La raison morphologique, 215-238.

Roizenzon, L. (1974). Mnogopristavochnye glagoly v russkom i drugikh slavianskikh iazykakh [Multiprefixal verbs in Russian and other Slavonic languages]. Samarkand: Publ. Samarkandskogo gosudarstwiennogo universiteta.

Skarżyński, M. (1999). Powstanie i rozwój polskiego słowotwórstwa opisowego. Kraków: Wydawnictwo Uniwersytetu Jagiellońskiego.

Skarżyński, M. (ed.). (2003a). Stowotwórstwo gniazdowe: historia, metoda, zastosowania. Kraków: Księgarnia Akademicka.

Skarżyński, M. (2003b). Słowotwórcze gniazda odczasownikowe (charakterystyka wstępna). In: M. Skarżyński (ed.). Stowotwórstwo gniazdowe: historia, metoda, zastosowania (pp. 137-155). Kraków: Księgarnia Akademicka.

Skarżyński, M. (2004). Wstęp. In: Stownik gniazd słowotwórczych współczesnego języka polskiego. T. III Gniazda odczasownikowe (pp. VII-XIV). Kraków: Towarzystwo Wydawnicze „Historia Iagellonica". 
Stankiewicz, J. (2006). Wymiana prefiksów w derywacji odczasownikowej (na materiale gniazdowym). Poradnik Językowy, 10, 119-126.

Śmiech, W. (1986). Derywacja prefiksalna czasowników polskich. Wrocław : Zakład Narodowy im. Ossolińskich.

Waszakowa, K. (1991). Derywacja sufiksalna, paradygmatyczna czy ujemna. Polonica, 16, (101-110).

Waszakowa, K. (1993). Stowotwórstwo współczesnego języka polskiego. Rzeczowniki z formantami paradygmatycznymi. Warszawa: Wydawnictwa Uniwersytetu Warszawskiego.

Wiśnicki, M. (2006). O derywacji prefiksalno-wymiennej w słowotwórczych gniazdach rzeczowników i przymiotników. Poradnik Językowy, 10, 126-133.

Wiśnicki, M. (2010). Derywacja prefiksalna wymienna czasowników polskich (w świetle danych gniazdowych). $\mathrm{PhD}$ thesis, typewritten. Warszawa.

Wróbel, H. (1998) Czasownik. In: R. Grzegorczykowa, R. Laskowski, H. Wróbel (eds.), Gramatyka współczesnego języka polskiego. Morfologia (pp. 536-583). Warszawa: PWN.

\section{RÉSUMÉ}

La technique de construction de mots que nous nous proposons d'étudier est une dérivation de verbes qui se fait par échange de préfixes. Elle fut découverte et décrite en 2006 par Joanna Stankiewicz, sa théorie fut développée en 2009 par Michał Wiśnicki pour le polonais et en 2010 par Wanda Fijałkowska pour le français. Les deux langues utilisent différentes paires de préfixes, p.ex. en français - embrigader - débrigader, décharger - recharger, congeler-surgeler, exploser - imploser et en polonais - narysować - odrysować, ugotować - nagotować, zadzwonić oddzwonić, zbudować - dobudować, zbroić - nabroić. La motivation sémantique des verbes cités comme seconds dans chaque paire devient évidente si nous nous servons de la paraphrase (selon la définition de Jadwiga Puzynina). La paraphrase correcte d'un dérivé doit contenir la base avec son préfixe. Ce type de paraphrase n'est pas utilisé par les chercheurs qui étudient le français; or, elle peut très bien servir à décrire la dérivation dans cette langue. Du point de vue logique, l'action décrite par le verbe dérivé ne peut avoir lieu avant celle qu'exprime le verbe de base: on ne peut odkręcić (dévisser) qch. qui n'est pas przykręcone (vissé), ni rozbudować (étendre) un bâtiment qui n'a pas encore été zbudowany (construit). Sinon, on ne pourrait expliquer pourquoi les préfixes des verbes comme débrigader, recharger, surgeler, odrysować, oddzwonić, nabroić sont spécifiques et ne furent pas simplement ajoutés aux verbes non munis d'un autre préfixe. Parmi les verbes accomplis polonais et français, on trouve des modèles productifs qui leur sont communs, p.ex. les deux langues forment des dérivés annulatifs (skręcić - rozkręcić, emballer - déballer), transformatifs (zbudować-przebudować, encoder-transcoder), de changement de direction (dobudować - nadbudować, exploser-imploser) et effectifs (umyć - domyć, congeler-surgeler). Les analyses menées ici constituent un premier pas pour prouver que cette technique de construction de mots a un caractère universel.

Mots-clés : construction de mots, préfixe, échange de préfixes, motivation, paraphrase

\section{ABSTRACT}

The word-formation technique to be tested is the derivation of verbs by exchange of prefixes, discovered and defined in 2006 by Joanna Stankiewicz, developed in 2006 and 2009 by Michat Wiśnicki for Polish, and in 2010 by Wanda Fijałkowska for French. In each language different prefixal pairs are used. The semantic motivation becomes obvious if we resort to word-formation 
paraphrases (as defined by Jadwiga Puzynina). A correctly formulated paraphrase of a derived word has to contain on the surface the original prefix - that of the base. This kind of paraphrases is not employed by French morphologists, but is applicable to studies on the French language. Logically, the activity named by the derived verb cannot occur before the activity named by its base is terminated: we cannot unscrew something that is not screwed, or extend a building that is not built yet. Otherwise, we cannot explain that the prefixes in such verbs as débrigader, recharger, surgeler, odrysować, oddzwonić, nabroić are unique and not simply added one to another. In spite of the differences between Polish and French accomplished verbs, common and productive patterns appear - for example, in both languages annulative, transformative, directional and effective verbs are created. The analyses that we have carried out are the first step towards proving the universality of this word-formation technique.

Keywords: word-formation, prefix, exchange of prefixes, motivation, paraphrase

\begin{abstract}
ABSTRAKT
Technika słowotwórcza, którą zbadano, to derywacja czasowników, dokonująca się przez wymianę prefiksów. Została ona odkryta i zdefiniowana w 2006 roku przez Joannę Stankiewicz, jej teoria została rozwinięta w 2006 i 2009 roku przez Michała Wiśnickiego dla języka polskiego oraz w 2010 roku przez Wandę Fijałkowską dla języka francuskiego. W obu językach używane są różne pary przedrostków. Motywacja semantyczna drugich członów tych par werbalnych staje się oczywista, jeśli posłużymy się parafrazą słowotwórczą (wg definicji Jadwigi Puzyniny). Poprawnie sformułowana parafraza derywatu powinna zawierać prefiks podstawy. Ten rodzaj parafraz nie bywa stosowany przez morfologów badających język francuski, lecz może znaleźć zastosowanie w studiach nad tym językiem. Z punktu widzenia logicznego czynność określona czasownikiem pochodnym nie może wydarzyć się wcześniej, niż zakończy się czynność wyrażona czasownikiem bazowym: nie można odkręcić czegoś, co nie jest przykręcone, ani rozbudować budynku, który nie został jeszcze zbudowany. W przeciwnym razie nie można wytłumaczyć, że przedrostki w takich czasownikach, jak: débrigader, recharger, surgeler, odrysować, oddzwonić, nabroić są specyficzne, i nie zostały po prostu dodane do podstaw bezprefiksalnych. Pomimo różnic między polskimi i francuskimi czasownikami dokonanymi pojawiają się wspólne dla nich produktywne wzorce, np. tworzone są derywaty anulatywne, transformatywne, kierunkowe i efektywne. Przeprowadzone analizy stanowią pierwszy krok w stronę dowodu, że opisywana technika słowotwórcza jest uniwersalna.
\end{abstract}

Słowa kluczowe: słowotwórstwo, prefiks, wymiana prefiksów, motywacja, parafraza 